\title{
Optimal realization of the transposition maps
}

\author{
F. Buscemi, G. M. D'Ariano, P. Perinotti, and M. F. Sacchi \\ Theoretical Quantum Optics Group \\ Università degli Studi di Pavia and INFM Unità di Pavia \\ via A. Bassi 6, I-27100 Pavia, Italy
}

\begin{abstract}
We solve the problem of achieving the optimal physical approximation of the transposition for pure states of arbitrary quantum systems for finite and infinite dimensions. A unitary realization is also given for any finite dimension, which provides the optimal quantum cloning map of the ancilla as well.
\end{abstract}

\section{Introduction}

The formulation of no-go theorems in quantum information has given new insight in the structure of the quantum theory itself. The most relevant examples are given by the no-cloning [1,2], no-broadcasting [3], no-deleting [4] theorems, along with the impossibility of measuring the wave-function of a single system [5], and the debated quantum bit-commitment [6].

A transformation which is not allowed by quantum mechanics naturally poses the problem of investigating about the best approximation that one can achieve in principle. Remarkably, the optimal approximation of a forbidden transformation may be related to the optimal procedure to perform some information tasks. For example, universal cloning is deeply related to the optimal eavesdropping in cryptographic channels [7]. Moreover, one can infer from the structure of the optimal approximation of unphysical maps a number of properties about different topics in quantum mechanics, such as state estimation [8] and signaling [9].

In this Letter we provide the optimal approximation of the transposition for pure states both in finite and infinite dimensional Hilbert spaces. Transposition - in particular, partial transposition — plays a major role in separability criteria for bipartite states [10]. It is the simplest example of a positive map, which is not completely positive. In fact, such a map is antilinear and for qubits it is related to the more familiar $U$-not transformation that maps any arbitrary 
state to its orthogonal. Of course, for systems with higher dimension the $U$-not map is not uniquely defined, because more than one state is orthogonal to a given one, whereas the transposition map depends on a choice of basis.

The paper is organized as follows. In Sec. II we derive the optimal transposition map for pure states in terms of fidelity for the case of arbitrary finite dimensional Hilbert spaces. The derivation is obtained by means of group representation theory. In Sec. III we give explicitly a unitary realization of the optimal map for any dimension. Such a unitary realization provides also the optimal quantum cloning from one to two copies. This result proves that the recent experimental scheme proposed in Ref. [11], where the optimal $U$-not and two optimal clones are created for qubits simultaneously by the same machine, can be generalized to quantum systems with arbitrary dimension. Section III is devoted to the continuous variables case. Similarly, as for the cloning map, one has to restrict the covariance group under which the map is universal. We consider the case of Weyl-Heisenberg group, which provides the optimal transposition map for coherent and squeezed states.

\section{Optimal transposition for finite dimension}

It is well known that the transposition map

$$
\rho \longmapsto \rho^{T}
$$

is not physical since it is not completely positive (CP). As stated in the introduction the problem naturally arises to find the optimal physical. i.e. CP map $\mathcal{M}$ whose output has maximal fidelity with the transposed input. We consider pure input states, for which the fidelity writes

$$
F=\operatorname{Tr}\left[\rho^{T} \mathcal{M}(\rho)\right]
$$

We settle here some useful notation that will be used along the paper. A generic vector in the bipartite Hilbert space $\mathcal{H} \otimes \mathcal{H}$, with $\operatorname{dim}(\mathcal{H})=d$, can be expanded on a fixed factorized orthonormal basis as $\sum_{i, j=1}^{d} \Psi_{i j}|i\rangle|j\rangle$. This naturally defines a one to one correspondence between vectors in $\mathcal{H} \otimes \mathcal{H}$ and linear operators in $\mathcal{L}(\mathcal{H})$. The operator $\Psi=\sum_{i j} \Psi_{i j}|i\rangle\langle j|$ can be used to label the state as follows

$$
|\Psi\rangle\rangle=\sum_{i, j=1}^{d} \Psi_{i j}|i\rangle|j\rangle .
$$

In this framework one can easily verify the useful identities [12] 


$$
\begin{aligned}
& \left.A \otimes C|B\rangle\rangle=\left|A B C^{T}\right\rangle\right\rangle, \\
& \operatorname{Tr}_{1}[|A\rangle\rangle\langle\langle B|]=A^{T} B^{*}, \\
& \operatorname{Tr}_{2}[|A\rangle\rangle\langle\langle B|]=A B^{\dagger},
\end{aligned}
$$

where $T$ and $*$ denote transposition and complex conjugation with respect to the orthonormal basis $\{|i\rangle\}$. The set of possible maps $\mathcal{M}$ can be parametrized using the isomorphism [13] between CP maps and positive operators

$$
\begin{aligned}
& \left.R_{\mathcal{M}}=\mathcal{M} \otimes \mathcal{I}|I\rangle\right\rangle\langle\langle I|, \\
& \mathcal{M}(\rho)=\operatorname{Tr}_{2}\left[\left(I \otimes \rho^{T}\right) R_{\mathcal{M}}\right] .
\end{aligned}
$$

Using Eq. (5), the expression for the fidelity writes

$$
F=\operatorname{Tr}\left[\left(\rho^{T} \otimes \rho^{T}\right) R_{\mathcal{M}}\right]
$$

where $R_{\mathcal{M}}$ is a positive operator that satisfies the constraint

$$
\operatorname{Tr}_{1}\left[R_{\mathcal{M}}\right]=I_{2}
$$

because of the trace-preserving condition of the map $\mathcal{M}$. We pose the further constraint that $\mathcal{M}$ is universal, namely that it is covariant under the action of the group $\mathrm{SU}(d)$. On physical grounds this means that we restrict attention to maps that equally well approximate the transposition, independently of the input pure state. The covariance property for the transposition map reads

$$
\mathcal{M}\left(U \rho U^{\dagger}\right)=U^{*} \mathcal{M}(\rho) U^{T}, \quad \forall U \in \mathrm{SU}(d)
$$

and this is equivalent to the following condition on $R_{\mathcal{M}}[14]$

$$
\left(U^{*} \otimes U^{*}\right) R_{\mathcal{M}}\left(U^{T} \otimes U^{T}\right)=R_{\mathcal{M}} .
$$

Since for $\mathrm{SU}(d)$ the representation $U^{*} \otimes U^{*}$ can be decomposed into two inequivalent irreducible representations supported by the totally symmetric and totally antisymmetric subspaces of $\mathcal{H} \otimes \mathcal{H}$, according to Schur's lemma, condition (9) implies the following form for $R_{\mathcal{M}}$

$$
R_{\mathcal{M}}=c_{A} P_{A}+c_{s} P_{S}
$$

The operators $P_{S}$ and $P_{A}$ are the projectors on the totally symmetric and totally antisymmetric spaces, respectively, and can be written as

$$
\begin{aligned}
& P_{S}=\frac{1}{2}(I+E), \\
& P_{A}=\frac{1}{2}(I-E),
\end{aligned}
$$


where $E$ denotes the swap operator on $\mathcal{H} \otimes \mathcal{H}$, namely $E|\phi\rangle|\psi\rangle=|\psi\rangle|\phi\rangle$ for all $\phi, \psi \in \mathcal{H}$. The trace-preserving condition in Eq. (7), along with the positivity constraint rewrite

$$
\begin{aligned}
& c_{S} \frac{d+1}{2}+c_{A} \frac{d-1}{2}=1, \\
& c_{S}, c_{A} \geq 0 .
\end{aligned}
$$

Due to the covariance condition, the fidelity $\mathrm{F}$ is independent of the input state $\rho$. Using Eq. (10) one has

$$
F=c_{S} \operatorname{Tr}\left[\left(\rho^{T} \otimes \rho^{T}\right) P_{S}\right]+c_{A} \operatorname{Tr}\left[\left(\rho^{T} \otimes \rho^{T}\right) P_{A}\right]=c_{S} .
$$

Upon maximizing $c_{S}$ with the constraints in Eq. (12), the optimal map is obtained for

$$
c_{S}=\frac{2}{d+1}, \quad c_{A}=0 .
$$

Correspondingly, one has $R_{\mathcal{M}}=\frac{2}{d+1} P_{S}$, and hence

$$
\mathcal{M}(\rho)=\frac{2}{d+1} \operatorname{Tr}_{2}\left[\left(I \otimes \rho^{T}\right) P_{S}\right]=\frac{1}{d+1}\left(I+\rho^{T}\right) .
$$

The optimal fidelity is then given by

$$
F=\frac{2}{d+1} \operatorname{Tr}\left[\left(\rho^{T} \otimes \rho^{T}\right) P_{S}\right]=\frac{2}{d+1} .
$$

The state in Eq. (15) coincides with the anticlone state of Ref. [15] for the universal cloning machine from one to two copies. Moreover, the map $\mathcal{M}$ is the same as the "structural physical approximation" of the transposition of Ref. [16]. Here, we proved the optimality of $\mathcal{M}$ without assumptions, thus also showing that the anticlone corresponds to the optimal transposed state.

A Kraus decomposition of the map $\mathcal{M}$ can be obtained by diagonalizing $R_{\mathcal{M}}$ as follows

$$
\begin{aligned}
& R_{\mathcal{M}}=\frac{1}{d+1}(I+E)=\frac{1}{d+1} \sum_{m n}(|m\rangle\langle m|\otimes| n\rangle\langle n|+| m\rangle\langle n|\otimes| n\rangle\langle m|) \\
& \left.\left.=\frac{1}{2(d+1)} \sum_{m n}(|m n\rangle\rangle+|n m\rangle\right\rangle\right)\left(\langle \langle m n | + \langle \langle n m | ) = \sum _ { m n } | M _ { m n } ^ { S } \rangle \rangle \left\langle\left\langle M_{m n}^{S}\right|,\right.\right.
\end{aligned}
$$

where $M_{m n}^{S}=\frac{1}{\sqrt{2(d+1)}}(|m\rangle\langle n|+| n\rangle\langle m|)$. The Kraus decomposition is then given by

$$
\mathcal{M}(\rho)=\sum_{m n} M_{m n}^{S} \rho M_{m n}^{S}
$$


A Stinespring form of the map $\mathcal{M}$ can be written for an ancilla in the Hilbert space $\mathcal{H}^{\otimes 2}$ as follows

$$
\mathcal{M}(\rho)=\operatorname{Tr}_{23}\left[V \rho V^{\dagger}\right]
$$

where $V$ denotes the isometry

$$
\left.V=\sum_{m n} M_{m n}^{S} \otimes|m n\rangle\right\rangle_{23}
$$

We can verify that $V$ is also an isometric extension for the realization of the optimal universal cloning from 1 to 2 copies. In fact, upon tracing out the system 1, one obtains the optimal cloning map in the Werner expression [17]

$$
\mathcal{C}(\rho)=\operatorname{Tr}_{1}\left[V \rho V^{\dagger}\right]=\frac{2}{d+1} P_{S_{23}}\left(I_{2} \otimes \rho_{3}\right) P_{S_{23}}
$$

This result also shows that the recent experimental scheme proposed in Ref. [11], where the optimal $U$-not and two optimal clones are created for qubits simultaneously by the same machine, can be generalized to quantum systems with arbitrary dimension. Notice that the cloning map is basis independent, whereas the transposition map depends on the choice of the basis, which is reflected by the particular Stinespring extension.

In the following we explicitly derive a unitary realization of the optimal map in Eq. (15).

\section{Unitary realization}

The isometry in Eq. (20) provides the optimal universal transposition map or the optimal universal cloning from one to two copies, by tracing over the ancilla spaces 2 and 3 or the input space 1, respectively. Starting from the isometry $V$, we look for a unitary interaction $U$ between the system and a fixed preparation of the ancilla that dilates $V$. For the explicit construction of the unitary dilation $U$, we will follow the general framework of Ref. [18].

First, notice that we can rewrite Eq. (20) as

$$
\begin{aligned}
V & =\sqrt{\frac{2}{d+1}} \sum_{m, n=0}^{d-1}|m\rangle \frac{|m\rangle|n\rangle+|n\rangle|m\rangle}{2}\langle n| \\
& =\sqrt{\frac{2}{d+1}} \sum_{m, q=0}^{d-1}|m\rangle \frac{|m\rangle|m \oplus q\rangle+|m \oplus q\rangle|m\rangle}{2}\langle m \oplus q| \\
& =\sqrt{\frac{2}{d+1}}\left(V_{0,0}+\frac{1}{\sqrt{2}} \sum_{q=1}^{d-1} V_{0, q}^{(S)}\right)
\end{aligned}
$$


where we defined the operators

$$
\begin{aligned}
V_{p, p} & =\sum_{k=0}^{d-1}|k\rangle|k \oplus p\rangle|k \oplus p\rangle\langle k \oplus p|, \\
V_{p, q}^{(S)} & =\frac{1}{\sqrt{2}} \sum_{k=0}^{d-1}|k\rangle(|k \oplus p\rangle|k \oplus q\rangle+|k \oplus q\rangle|k \oplus p\rangle)\langle k \oplus q|,
\end{aligned}
$$

with $p \neq q$ and $p, q=0, \ldots, d-1$. In order to construct a unitary realization $U$, we also define

$$
V_{p, q}^{(A)}=\frac{1}{\sqrt{2}} \sum_{k=0}^{d-1}|k\rangle(|k \oplus p\rangle|k \oplus q\rangle-|k \oplus q\rangle|k \oplus p\rangle)\langle k \oplus q| .
$$

One can easily verify that

$$
\begin{aligned}
& V_{p, p}^{\dagger} V_{q, r}^{(S)}=V_{p, p}^{\dagger} V_{s, t}^{(A)}=V_{q, r}^{(S) \dagger} V_{s, t}^{(A)}=0, \quad \forall p, q, r, s, t=0, \ldots, d-1 \\
& V_{p_{1}, p_{1}}^{\dagger} V_{p_{2}, p_{2}}=\delta_{p_{1}, p_{2}} I_{\mathcal{H}}, \quad \forall p_{1}, p_{2}, \\
& V_{q_{1}, r_{1}}^{(S) \dagger} V_{q_{2}, r_{2}}^{(S)}=\delta_{q_{1}, q_{2}} \delta_{r_{1}, r_{2}} I_{\mathcal{H}}, \quad \forall q_{1}<r_{1}, q_{2}<r_{2}, \\
& V_{s_{1}, t_{1}}^{(A) \dagger} V_{s_{2}, t_{2}}^{(A)}=\delta_{s 1, t_{2}} \delta_{s_{1}, t_{2}} I_{\mathcal{H}}, \quad \forall s_{1}<t_{1}, s_{2}<t_{2},
\end{aligned}
$$

namely, the three sets $\left\{V_{p, p}\right\},\left\{V_{p, q}^{(S)}\right\}_{p<q}$ and $\left\{V_{p, q}^{(A)}\right\}_{p<q}$ are orthogonal sets of orthogonal isometries. Hence, the following operator

$U=\sum_{p=0}^{d-1} V_{p, p} \otimes\langle p|\langle p|+\sum_{\substack{p, q=0 \\ p<q}}^{d-1} V_{p, q}^{(S)} \otimes \frac{\langle p|\langle q|+\langle q|\langle p|}{\sqrt{2}}+\sum_{\substack{p, q=0 \\ p<q}}^{d-1} V_{p, q}^{(A)} \otimes \frac{\langle p|\langle q|-\langle q|\langle p|}{\sqrt{2}}$

satisfies the unitary condition

$$
U^{\dagger} U=U U^{\dagger}=I_{\mathcal{H}} \otimes\left(P_{S_{23}}+P_{A_{23}}\right)=I_{\mathcal{H} \otimes 3}
$$

The optimal universal transposition map can be obtained as follows

$$
\mathcal{M}(\rho)=\operatorname{Tr}_{2,3}\left[U(\rho \otimes|\phi\rangle\rangle\langle\langle\phi|) U^{\dagger}\right]
$$

where $|\phi\rangle\rangle \in \mathcal{H}^{\otimes 2}$ is the fixed normalized totally symmetric ancilla state

$$
|\phi\rangle\rangle=\sqrt{\frac{2}{d+1}} P_{S_{23}} \sum_{r=0}^{d-1}|0\rangle_{2}|r\rangle_{3} .
$$

As noted above, the unitary $U$ provides the optimal universal cloning as well, namely one has

$$
\mathcal{C}(\rho)=\operatorname{Tr}_{1}\left[U(\rho \otimes|\phi\rangle\rangle\langle\langle\phi|) U^{\dagger}\right]
$$


For qubits, i.e. $d=2$, we obtain the network model of Ref. [19], with

$$
U=\left(\begin{array}{llllllll}
1 & 0 & 0 & 0 & 0 & 0 & 0 & 0 \\
0 & 0 & 0 & 0 & 0 & 1 & 0 & 0 \\
0 & 0 & 0 & 0 & 0 & 0 & 1 & 0 \\
0 & 0 & 0 & 0 & 0 & 0 & 0 & 1 \\
0 & 0 & 0 & 1 & 0 & 0 & 0 & 0 \\
0 & 0 & 1 & 0 & 0 & 0 & 0 & 0 \\
0 & 1 & 0 & 0 & 0 & 0 & 0 & 0 \\
0 & 0 & 0 & 0 & 1 & 0 & 0 & 0
\end{array}\right),
$$

and $|\phi\rangle\rangle=\frac{1}{\sqrt{6}}\left(2|0\rangle_{2}|0\rangle_{3}+|0\rangle_{2}|1\rangle_{3}+|1\rangle_{2}|0\rangle_{3}\right)$.

\section{Continuous variables optimal transposition}

In the limit of dimension $d \rightarrow \infty$, the fidelity $F$ for the universal transposition map goes to zero. However, as regards infinite dimensional systems one can look for transposition maps that are not universal, but covariant just for a group with reduced symmetry. The typical covariance group for infinite dimensional quantum systems is the Weyl-Heisenberg group, in the representation of displacement operators $D(\alpha)=\exp \left(\alpha a^{\dagger}-\alpha^{*} a\right)$, with $\alpha \in \mathbb{C}$, and $a$ and

$a^{\dagger}$ being the annihilation and creation operators. The covariant transposition map acts with the same fidelity over any state obtained from a given pure state by application of the displacement operator with arbitrary amplitude. Such a covariance condition reads

$$
\mathcal{M}\left(D(\alpha) \rho D^{\dagger}(\alpha)\right)=D^{*}(\alpha) \mathcal{M}(\rho) D^{T}(\alpha), \quad \forall \alpha \in \mathbb{C}
$$

which rewrites for the operator $R_{\mathcal{M}}$ as follows

$$
\left[D^{*}(\alpha) \otimes D^{*}(\alpha), R_{\mathcal{M}}\right]=0, \quad \forall \alpha \in \mathbb{C} .
$$

The operator $R_{\mathcal{M}}$ can be expanded on the basis of displacement operators, which are a spanning set for Hilbert-Schmidt operators on $\mathcal{H}$, namely

$$
R_{\mathcal{M}}=\int_{\mathbb{C}} \frac{\mathrm{d}^{2} \alpha}{\pi} \int_{\mathbb{C}} \frac{\mathrm{d}^{2} \beta}{\pi} r(\alpha, \beta) D(\alpha) \otimes D(\beta) .
$$

The condition in Eq. (33) is then equivalent to 


$$
\begin{aligned}
& \int_{\mathbb{C}} \frac{\mathrm{d}^{2} \alpha}{\pi} \int_{\mathbb{C}} \frac{\mathrm{d}^{2} \beta}{\pi} r(\alpha, \beta) \mathrm{e}^{\gamma(\alpha+\beta)-\gamma^{*}\left(\alpha^{*}+\beta^{*}\right)} D(\alpha) \otimes D(\beta)= \\
& \int_{\mathbb{C}} \frac{\mathrm{d}^{2} \alpha}{\pi} \int_{\mathbb{C}} \frac{\mathrm{d}^{2} \beta}{\pi} r(\alpha, \beta) D(\alpha) \otimes D(\beta), \quad \forall \gamma \in \mathbb{C},
\end{aligned}
$$

and this is possible for $r(\alpha, \beta)=\pi r(\alpha) \delta^{2}(\alpha+\beta)$, with $r(\alpha)$ complex function of $\alpha$, thus giving

$$
R_{\mathcal{M}}=\int_{\mathbb{C}} \frac{\mathrm{d}^{2} \alpha}{\pi} r(\alpha) D(\alpha) \otimes D^{\dagger}(\alpha)
$$

The trace-preserving condition $\operatorname{Tr}_{1}\left[R_{\mathcal{M}}\right]=I_{2}$ corresponds to $r(0)=1$. Upon introducing the $50 / 50$ beam splitter operator $V=\exp \left[\frac{\pi}{4}\left(a^{\dagger} b-a b^{\dagger}\right)\right]$, we can write

$$
R_{\mathcal{M}}=V \int_{\mathbb{C}} \frac{\mathrm{d}^{2} \alpha}{\pi} r(\alpha)[D(\sqrt{2} \alpha) \otimes I] V^{\dagger} \equiv \frac{1}{2} V^{\dagger}(\xi \otimes \mathbb{1}) V,
$$

and $R_{\mathcal{M}}$ is positive if and only if the following operator

$$
\xi \equiv \int_{\mathbb{C}} \frac{\mathrm{d}^{2} \alpha}{\pi} r\left(\frac{\alpha}{\sqrt{2}}\right) D(\alpha)
$$

is positive. Since $r(0)=1$, then $\operatorname{Tr}[\xi]=1$, namely $\xi$ is a state. For the covariance condition in Eq. (33), the fidelity of the map with the state $\left[D(\alpha) \rho D^{\dagger}(\alpha)\right]^{\tau}$ is independent of $\alpha$ and just depends on the seed $\rho$. One has

$$
F=\frac{1}{2} \operatorname{Tr}\left[\left(\rho^{\tau} \otimes \rho^{\tau}\right) V(\xi \otimes \mathbb{1}) V^{\dagger}\right]
$$

Equation (39) is linear in $\xi$, which lies in a convex set. The maximum fidelity is then achieved by a pure state $\xi=|\chi\rangle\langle\chi|$, and the optimal map is given by

$$
R_{\mathcal{M}}=\frac{1}{2} V(|\chi\rangle\langle\chi| \otimes \mathbb{1}) V^{\dagger}
$$

The vector $|\chi\rangle$ can be determined as the eigenvector corresponding to the maximum eigenvalue of the state

$$
\operatorname{Tr}_{2}\left[V^{\dagger}\left(\rho^{\tau} \otimes \rho^{\tau}\right) V\right]
$$

The explicit form of the map acting on a general state $\sigma$ is given by

$$
\mathcal{M}(\sigma)=\frac{1}{2} \operatorname{Tr}_{2}\left[\left(\mathbb{1} \otimes \sigma^{\tau}\right) V(|\chi\rangle\langle\chi| \otimes \mathbb{1}) V^{\dagger}\right]
$$

and provides the optimal transposition for any pure state. We remind that $|\chi\rangle$ depends on the seed state $\rho$. Notice that for coherent states, namely for $\rho=|0\rangle\langle 0|$, the optimal transposed state can be obtained as the anticlone from the optimal covariant cloning from one to two copies [20], with optimal fidelity $F=1 / 2$, generalizing the result for the finite dimensional case. 


\section{Acknowledgments}

This work has been sponsored by INFM through the project PRA-2002CLON, and by EEC and MIUR through the cosponsored ATESIT project IST-2000-29681 and Cofinanziamento 2002.

\section{References}

[1] W. K. Wootters and W. H. Zurek, Nature 299, 802 (1982).

[2] H. P. Yuen, Phys. Lett. A 113, 405 (1986).

[3] H. Barnum, G. M. Caves, C. A. Fuchs, R. Josza, and B. Schumacher, Phys. Rev. Lett. 76, 2818 (1996).

[4] A. K. Pati and S. L. Braunstein, Nature 404, 164 (2000).

[5] G. M. D'Ariano and H. P. Yuen, Phys. Rev. Lett. 76, 2832 (1996).

[6] D. Mayers, Phys. Rev. Lett. 78, 3414 (1997); H. K. Lo and H. F. Chau, Phys. Rev. Lett. 78, 3410 (1997); H. K. Lo, Phys. Rev. A 56, 1154 (1997); H. P. Yuen, quant-ph/0210206; G. M. D'Ariano, quant-ph/0209149.

[7] C. Fuchs, N. Gisin, R. Griffiths, C.-S. Niu and A. Peres, Phys. Rev. A 56, 1163 (1997); D. Bruß, Phys. Rev. Lett. 81, 3018 (1998); H. Bechmann-Pasquinucci and N. Gisin, Phys. Rev. A 59, 4238 (1999); D. Bruss and C. Macchiavello, Phys. Rev. Lett. 88, 127901 (2002).

[8] D. Bruss, A. Ekert, and C. Macchiavello, Phys. Rev. Lett. 81, 2598 (1998); D. Bruss and C. Macchiavello, Phys. Lett. A 253, 249 (1999).

[9] N. Gisin, Phys. Lett. A 242, 1 (1998); L. Hardy and D. D. Song, Phys. Lett. A 259, 331 (1999); D. Bruss, G. M. D'Ariano, C. Macchiavello, and M. F. Sacchi, Phys. Rev. A 62, 062302 (2000); C. Simon, V. Bužek, and N. Gisin, Phys. Rev. Lett. 87, 170405 (2001).

[10] A. Peres, Phys. Rev. Lett. 77, 1413 (1996); M. Horodecki, P. Horodecki, and R. Horodecki, Phys. Lett. A 223, 1 (1996).

[11] D. Pelliccia, V. Schettini, F. Sciarrino, C. Sias, and F. De Martini, quant$\mathrm{ph} / 0302087$.

[12] G. M. D'Ariano, P. Lo Presti, and M. F. Sacchi, Phys. Lett. A 272, 32 (2000).

[13] A. Jamiołkowski, Rep. Math. Phys. 3, 275 (1972).

[14] G. M. D'Ariano and P. Lo Presti, Phys. Rev. A 64, 042308 (2001).

[15] V. Bužek and M. Hillery, Phys. Rev. Lett. 81, 5003 (1998). 
[16] P. Horodecki, quant-ph/0111036 (2001).

[17] R. Werner, Phys. Rev. A 58, 1827 (1998).

[18] F. Buscemi, G. M. D'Ariano, and M. F. Sacchi, in preparation.

[19] V. Bužek, S. L. Braunstein, M. Hillery, and D. Bruss, Phys. Rev. A 56, 3446 (1997).

[20] N. J. Cerf and S. Iblisdir, Phys. Rev. A 64, 032307 (2001). 